\title{
Applicability of duplex real time and lateral flow strip reverse-transcription recombinase aided amplification assays for the detection of Enterovirus 71 and Coxsackievirus A16
}

Xin-na Li ${ }^{1+}$, Xin-xin Shen ${ }^{1 \dagger}$, Ming-hui $\mathrm{Li}^{2+}$, Ju-ju Qi ${ }^{1}$, Rui-huan Wang ${ }^{1}$, Qing-xia Duan ${ }^{1}$, Rui-qing Zhang ${ }^{1}$, Tao Fan ${ }^{1}$, Xue-ding Bai ${ }^{1}$, Guo-hao Fan', Yao Xie ${ }^{2^{*}}$ and Xue-jun Ma ${ }^{1^{*}}$

\begin{abstract}
Background: Enterovirus 71 (EV71) and coxsackievirus A16 (CA16) are the two main etiological agents of Hand, Foot and Mouth Disease (HFMD). Simple and rapid detection of EV71 and CA16 is critical in resource-limited settings.

Methods: Duplex real time reverse-transcription recombinase aided amplification (RT-RAA) assays incorporating competitive internal amplification controls (IAC) and visible RT-RAA assays combined with lateral flow strip (LFS) for detection of EV71 and CA16 were developed respectively. Duplex real time RT-RAA assays were performed at $42^{\circ} \mathrm{C}$ within 30 min using a portable real-time fluorescence detector, while LFS RT-RAA assays were performed at $42^{\circ} \mathrm{C}$ within 30 min in an incubator. Recombinant plasmids containing conserved VP1 genes were used to analyze the sensitivities of these two methods. A total of 445 clinical specimens from patients who were suspected of being infected with HFMD were used to evaluate the performance of the assays.

Results: The limit of detection (LoD) of the duplex real time RT-RAA for EV71 and CA16 was 47 copies and 38 copies per reaction, respectively. The LoD of the LFS RT-RAA for EV71 and CA16 were both 91 copies per reaction. There was no cross reactivity with other enteroviruses. Compared to reverse transcription-quantitative PCR (RTqP(R), the clinical diagnostic sensitivities of the duplex real time RT-RAA assay were $92.3 \%$ for EV71 and 99.0\% for CA16, and the clinical diagnostic specificities were 99.7 and 100\%, respectively. The clinical diagnostic sensitivities of the LFS RT-RAA assay were $90.1 \%$ for EV71 and $94.9 \%$ for CA16, and the clinical diagnostic specificities were 99.7 and $100 \%$, respectively.
\end{abstract}

Conclusions: The developed duplex real time RT-RAA and LFS RT-RAA assays for detection of EV71 and CA16 are potentially suitable in primary clinical settings.

Keywords: Enterovirus 71, Coxsackievirus A16, Hand foot and mouth disease, Duplex, Reverse-transcription recombinase aided amplification assays, Internal amplification controls (IAC), Lateral flow strip

\footnotetext{
* Correspondence: xieya000120184@sina.com; maxj@ivdc.chinacdc.cn

${ }^{+}$Xin-na Li, Xin-xin Shen and Ming-hui Li, the three authors contributed equally to this study.

${ }^{2}$ Department of hepatology Division 2, Beijing Ditan Hospital, Capital Medical University, Jing Shun Dong Jie 8\#, Beijing 100015, China

${ }^{1} \mathrm{NHC}$ Key Laboratory of Medical Virology and Viral Diseases, Chinese Center for Disease Control and Prevention, National Institute for Viral Disease

Control and Prevention, No.155 Changbai Road, Changping district, Beijing

102206, China
}

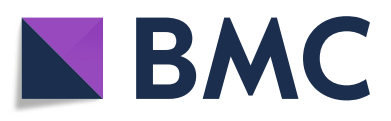

(c) The Author(s). 2019 Open Access This article is distributed under the terms of the Creative Commons Attribution 4.0 International License (http://creativecommons.org/licenses/by/4.0/), which permits unrestricted use, distribution, and reproduction in any medium, provided you give appropriate credit to the original author(s) and the source, provide a link to the Creative Commons license, and indicate if changes were made. The Creative Commons Public Domain Dedication waiver (http://creativecommons.org/publicdomain/zero/1.0/) applies to the data made available in this article, unless otherwise stated. 


\section{Background}

Hand, foot and mouth disease (HFMD) is a common acute infectious disease with typical rash distribution characteristics in the mouth, hands and feet, and occurs mainly in the children under 5 years of age. Enteroviruses such as enterovirus 71 (EV71), coxsackievirus A16 (CA16), coxsackievirus A6 (CA6) and coxsackievirus A10 (CA10) are the main pathogens causing the disease. According to previous monitoring reports, EV71 and CA16 have co-circulated as two most frequent EV types in causing repeated HFMD outbreak in different areas [1-6]. EV71-related HFMD can be accompanied by serious complications, such as myocarditis, pulmonary edema, aseptic meningitis, a proportion of which are fatal $[7,8]$, while CA16-related HFMD is usually mild and self-limiting.

Virus isolation, neutralization tests and nucleic acid amplification are commonly used for the detection and diagnosis of EV71 and CA16 [9, 10]. Virus isolation and neutralization are not rapid and accurate enough because of complex procedures and low specificities and sensitivities. Quantitative PCR (qPCR), reverse transcription-quantitative PCR (RT-qPCR) as a gold standard method is widely used to detect pathogens [11-17], since it is highly sensitive and specific analysis. Nevertheless, RT-qPCR protocols require specialized PCR machines and take more than $2 \mathrm{~h}$. In recent years, many isothermal methods have emerged, such as nucleic acid sequence based amplification [18, 19], loop mediated isothermal amplification [20, 21], and recombinase polymerase amplification [22]. These assays are performed at a constant temperature for less than $1 \mathrm{~h}$ with high sensitivities, specificities and do not require use of thermal cycler, which represents valuable alternatives to carry out simple and rapid pathogen detection.

Recombinase aided amplification (RAA) is an isothermal amplification technique and is performed at 37$42{ }^{\circ} \mathrm{C}$ for $30 \mathrm{~min}$. There are three main proteins in the RAA system: recombinase, single-stranded DNA binding protein (SSB) and DNA polymerase Klenow fragment. The amplification is initiated by a primer recombinase complex. The complex invades the DNA double strand at the homologues sequences of the primer, where SSB stabilizes the reaction. The polymerase is responsible for extension. The RAA assay can also use reverse transcriptase for the detection of RNA template. Real time detection of the RAA products can be achieved by adding exonuclease III (exo) and exo-probe. While visual detection can be realized by combining lateral flow strip (LFS) with RAA assay, making it an ideal technique for point-of-care testing [23-25]. The expected result of the positive reaction is clear colored test line and control line on the strip. The negative reaction does not generate a signal at the position of the test line (Fig. 1) [24].

Previous studies reported on the applications of RAA in the detection of salmonella [27] and RSV, CA6 and CA10 and HBV [28-30], but these methods did not use internal amplification control (IAC) or LFS. The objective of the research is to establish sensitive and rapid RAA assays for the detection of EV71 and CA16, including duplex real time RT-RAA assay containing IAC to reduce the false negative rates and LFS RT-RAA assay suitable for field detection in resource limited areas.

\section{Methods}

Clinical samples

HFMD clinical diagnostic criteria was referred to Handfoot-mouth disease diagnosis and treatment guidelines (2010 version) [31]. A total of 445 clinical samples from patients ( 7 months to 11 years of age) who were suspected of being infected with HFMD in Shandong province, Hebei province, and Hunan province in China were collected during the period from January 2016 to December 2016 for this study. Sample types included throat swabs $(n=76)$, anal swabs $(n=25)$ and stools $(n=344)$. Ethics approval was granted by the local ethics committee.

\section{Nucleic acid extraction}

Pre-treatment of clinical specimens was described previously [30]. According to the instructions recommended by the manufacturer, the total RNA was extracted from $200 \mu \mathrm{L}$ of sample preservation solutions or supernatants (fecal treatment fluid) using the Tian Long RNA extraction kit (Tian Long, China). The nucleic acid was eluted in $100 \mu$ Lof nuclease free water.

\section{Preparation of plasmid standards and IAC plasmids}

The cDNA of the viral protein 1 gene of EV71 or CA16 was cloned into the pClone007 vector, the standard recombinant plasmids with 10 -fold concentrations ranging from $10^{6}$ copies $/ \mu \mathrm{L}$ to $10^{\circ}$ copies $/ \mu \mathrm{L}$ were made and stored at $-80^{\circ} \mathrm{C}$ until used. The IAC templates were recombinant plasmids consisting of the IAC exo probe sequence, a short gene sequence of rose rosette virus [32], which replaced the corresponding probe sequences of EV71 and CA16, respectively (Fig. 2).

\section{Primers and probes design of RT-RAA assays for the detection of EV71 andCA16}

The VP1 genes of both EV71 and CA16 were chosen as the targets because VP1 was a specific region for enterovirus genotyping. All the available VP1 genes of EV71 and CA16 were downloaded from GenBank database. 


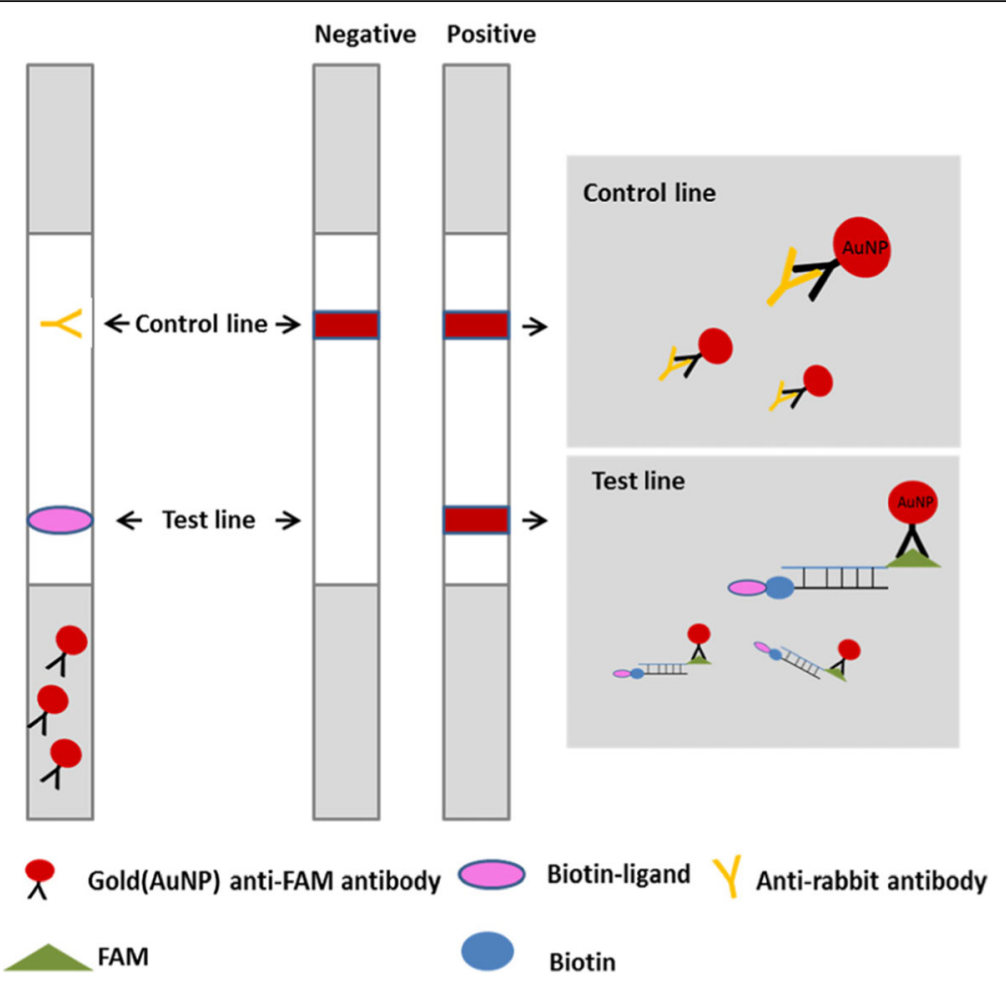

Fig. 1 Detection of the RAA amplicons by lateral flow strip. The sample pad contains gold-labeled anti-FAM antibodies, the test line was coated with biotin-ligands, and control line was coated with anti-rabbit antibodies. The double-labeled amplicons (FAM and biotin) were diffused through the chromatographic membrane, and when they diffused to the test line, the products were captured by the biotin -ligands, resulting in an appearance of red-pink color. Non-captured particles will be fixed at the control line by anti-rabbit antibodies. In the absence of target amplicons, color will appear at control line only [26]

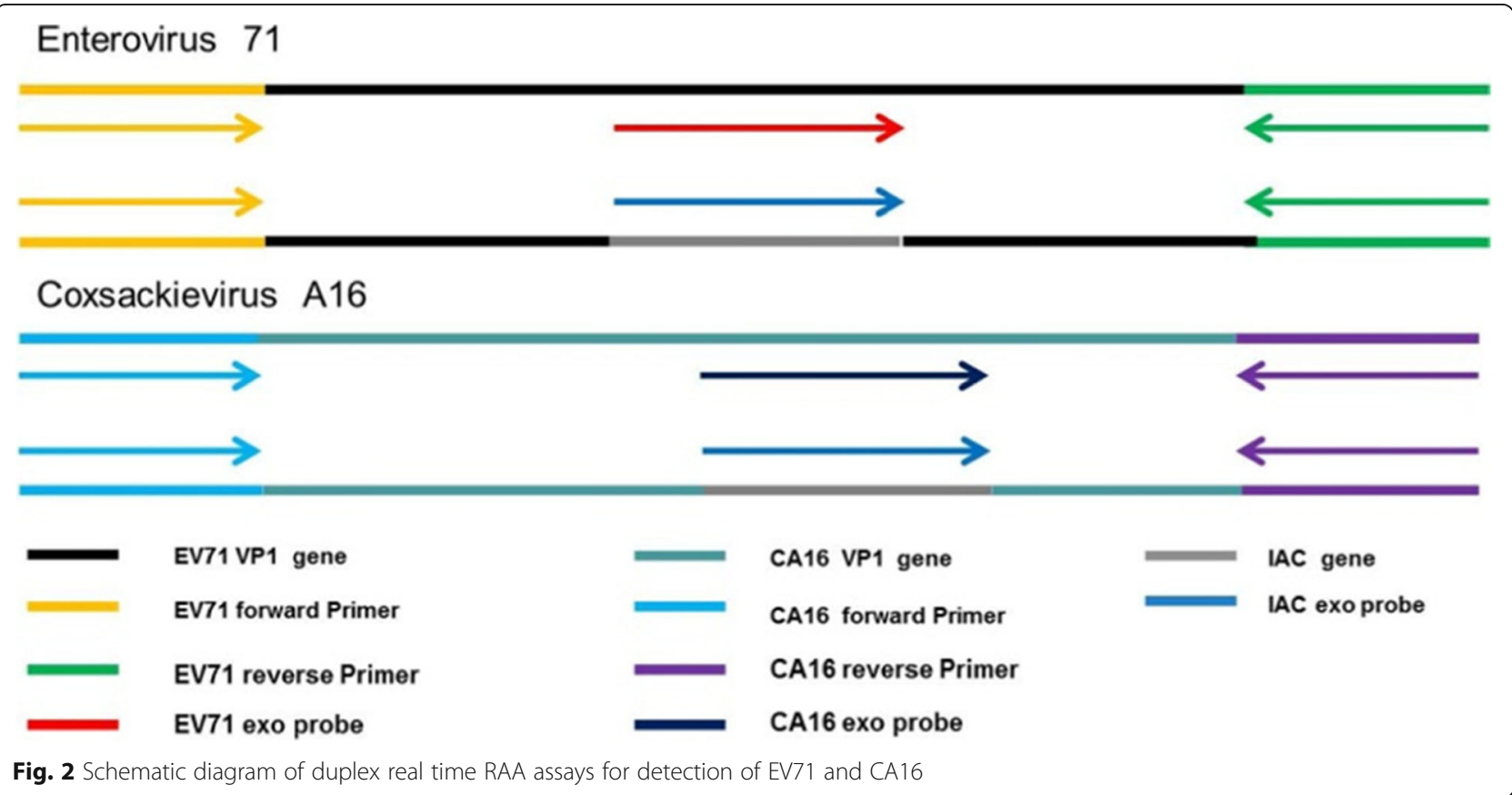


The sequences were aligned by Unipro UGENE. The primers were manually designed following the principle of RAA primer and probe design [33]: (1) Primers usually are 30-35 nucleotides long in length. (2) Long tracks of guanines at the $5^{\prime}$ end are avoided. (3) Guanines and cytidines at the $3^{\prime}$ end are preferred. (4) Probes are about 46-52 nucleotides long in length. (5) A DNA exo probe is used for real time assay, consisting of an oligonucleotide with homology to the target amplicon and an abasic nucleotide analogue (tetrahydrofuran residue or THF) flanked by a dT-fluorophore and a corresponding $\mathrm{dT}$-quencher group. In addition, probe is blocked from polymerase extension by C3-spacer. (6) A DNA probe is used for LFS assay, consisting of 5-FAM antigenic labeled upstream stretch (30 nucleotides) connected via THF spacer to an adjacent downstream oligonucleotide (15 nucleotides) carrying a C3-spacer. (7) Reverse primers used for LFS assay is labelled at its $5^{\prime}$ end with biotin. The target exo probes and IAC exo probe were modified with FAM and HEX fluorophores, respectively. All the primer and probe sequences are listed in Table 1. The primers and probes were synthesized by Sangon Biotech (Shanghai, China).

\section{Duplex real time RT-RAA assay}

The duplex real time RT-RAA assays were carried out using the exo-RT-RAA lyophilized kit (Qitian Bio-Tech, China). The total reaction volume was $50 \mu \mathrm{L}$ containing $420 \mathrm{nM}$ of RAA primers, $120 \mathrm{nM}$ of target exo probe for EV71, or $150 \mathrm{nM}$ of target exo probe for CA16, and 60 $\mathrm{nM}$ of IAC exo probe for IAC, and an IAC recombinant plasmid (50 copies per reaction for EV71 or100 copies per reaction for CA16, respectively), $14 \mathrm{mM}$ magnesium acetate and $2 \times$ buffer. The RT-RAA reagents were made in a master mix and were rehydrated pellets. Magnesium acetate and $5 \mu \mathrm{L}$ of template were added into the reaction tubes, subsequently, tubes were placed into B6100 Oscillation mixer (QT-RAA-B6100, Jiangsu Qitian BioTech Co. Ltd., China) and incubated for $4 \mathrm{~min}$, then mixed and centrifuged briefly, transferred to fluorescence detector (QT-RAA-1620, Jiangsu Qitian Bio-Tech Co. Ltd., China) at $42^{\circ} \mathrm{C}$ for $30 \mathrm{~min}$. The FAM channel was used to detect the amplification of the target gene (EV71 or CA16), and the HEX channel was used to detect the amplification of the IAC. Fluorescence data were normalized and baseline was adjusted using RAA 1620 software. External positive and negative controls were included to avoid false negative and positive results in each assay.

\section{LFS RT-RAA assay and optimization of the reaction time} LFS RT-RAA assays were performed using the nfo-RTRAA lyophilized kit (Qitian Bio-Tech, China). The total $50 \mu$ Lvolume containing $420 \mathrm{nM}$ of RAA primers, 120 $\mathrm{nM}$ of LF probe for EV71, $150 \mathrm{nM}$ of LF probe for CA16. To determine the optimal time of the LFS RTRAA assays, the tubes were incubated in the preequilibrated device at $42{ }^{\circ} \mathrm{C}$ for $10,20,30$ and $40 \mathrm{~min}$, respectively, using $1.0 \times 10^{2}$ copies of the recombinant plasmids as templates. After amplification, the RAA products were detected by lateral flow strip (Ustar Biotechnologies, Hangzhou, China) according to the

Table 1 List of primers used in the study for rapid detection of EV71 and CA16

\begin{tabular}{|c|c|c|c|c|}
\hline Assay & $\begin{array}{l}\text { Primer/ } \\
\text { probe }\end{array}$ & Sequence $5^{\prime}-3^{\prime}$ & $\begin{array}{l}\text { Genomic } \\
\text { Position }^{\text {a }}\end{array}$ & $\begin{array}{l}\text { Product } \\
\text { size }\end{array}$ \\
\hline \multirow{7}{*}{$\begin{array}{l}\text { Real time } \\
\text { RAA }\end{array}$} & EV71-exo-F & CCTGCGAGTGCTTACCAATGGTITATGACGG & $3026-3057$ & \multirow[t]{3}{*}{$199 \mathrm{bp}$} \\
\hline & EV71-exo-R & GTATCCACGCCCTGACGTGCTTCATTCTCAT & $3194-3224$ & \\
\hline & EV71-exo-P & $\begin{array}{l}\text { AACATGATGGGCACGTTCTCAGTGCGGAC-[FAM-dT]-[THF] -[BHQ-dT]-GGGGACCTCCAAGTC- } \\
\text { C3-spacer }\end{array}$ & $3119-3165$ & \\
\hline & CA16-exo-F & GCAAGTAGTCACAGATTAGGCACTGGTGTTGT & $2557-2588$ & \multirow[t]{4}{*}{$161 \mathrm{bp}$} \\
\hline & CA16-exo-R & GCACGGCTAAAGAAATTCCCAATGGCTGTC & $2688-2717$ & \\
\hline & CA16-exo-P & $\begin{array}{l}\text { GTGACAAGAATCTCATTGAGACKAGATG-[FAM-dT]-[THF] [BHQ-dT]-GTTGAACCATCACTCCA- } \\
\text { C3-spacer }\end{array}$ & $2633-2680$ & \\
\hline & IAC-P & $\begin{array}{l}\text { GTAAGGTGCTAGACTAAAATTGTTGGGACTT- [HEXdT]-G [THF]-A-[BHQ-dT]-CTCTGAAGTAAA } \\
\text { AGG-C3-spacer }\end{array}$ & & \\
\hline \multirow[t]{6}{*}{ LFS RAA } & EV71-LF-F & CCTGCGAGTGCTTACCAATGGTITATGACGG & $3026-3057$ & \multirow[t]{3}{*}{$199 \mathrm{bp}$} \\
\hline & EV 71-LF-R & Biotin-GTATCCACGCCCTGACGTGCTTCATTCTCAT & $3194-3224$ & \\
\hline & EV71-LF-P & FAM-AACATGATGGGCACGTTCTCAGTGCGGAC -[THF]-TGGGGACCTCCAAGTC-C3-spacer & $3119-3165$ & \\
\hline & CA16-LF-F & GCAAGTAGTCACAGATTAGGCACTGGTGTTGT & $2557-2588$ & \multirow[t]{3}{*}{$161 \mathrm{bp}$} \\
\hline & CA16-LF-R & Biotin-GCACGGCTAAAGAAATTCCCAATGGCTGTC & $2688-2717$ & \\
\hline & CA16-LF-P & FAM -GTGACAAGAATCTCATTGAGACKAGATG-[THF] -TGTTGAACCATCACTCCA-C3-spacer & $2633-2680$ & \\
\hline
\end{tabular}

${ }^{\mathrm{a} G e n o m e ~ p o s i t i o n ~ d e p e n d i n g ~ o n ~ E n t e r o v i r u s ~ A 71 ~ s t r a i n ~ H P ~(G e n B a n k ~ a c c e s s i o n ~ n o . K Y 074643.1) ~ a n d ~ C o x s a c k i e v i r u s ~ A 16 ~ i s o l a t e ~ Z J 10-48 ~(G e n B a n k ~ a c c e s s i o n ~}$ no. KC755235.1) 
instruction of manufacturer. The result was considered negative if only the control line was visible. The result was considered to be positive when both the control line and test line visible (Fig. 1).

\section{Analytical sensitivity and specificity of duplex real time RT-RAA and LFS RT-RAA assays}

The analytical sensitivity of real time RT-RAA and LFS RT-RAA assays for EV71 and CA16 were tested by using a serial dilution of recombinant plasmid standards ranging from $10^{6}$ to $10^{\circ}$ copies in eight replicates. The LoD of two assays were calculated using the probit regression analysis with the SPSS version 17.0. The analytical specificity of the RT-RAA assays for EV71 and CA16 was respectively tested by using 170 Non-EV71, non-CA16 enterovirus positive specimens (out of 445) in the study.

\section{Evaluation of duplex real time RT-RAA and LFS RT-RAA with clinical specimens}

The duplex real-time and LFS RT-RAA assays were assessed by using 445 clinical specimens. For the duplex real time RT-RAA assays, detection results were considered positive by simultaneously generating amplification curves from both clinical specimens and IAC. Detection was identified to be negative when there was only amplification of IAC. Neither IAC nor clinical specimens was amplified indicated invalid assay. For the LFS RT-RAA assays, if the control line and test line appeared simultaneously, the result was judged to be positive; if there was only control line, it was judged to be negative, if there was no control line and test line, it was judged to be invalid. The real time RT-qPCR assays for EV71, CA16 and other enteroviruses were carried out simultaneously as parallel tests [11]. The result was judged as positive when threshold cycle $(\mathrm{Ct})$ value was less than 35. Inconsistent detection results were further resolved using nested RT-PCR assays and sequencing [34]. The overall clinical performance was evaluated by calculating diagnostic sensitivity, diagnostic specificity, positive predictive value (PPV), negative predictive value (NPV), and Kappa value [35, 36].

\section{Results}

Analytical sensitivity and specificity of duplex real time RT-RAA assay

As shown in the Fig. 3, in the FAM channel, increase of fluorescence signal was observed from $1 \times 10^{6}$ to $1 \times 10^{0}$ copies/reaction at $42{ }^{\circ} \mathrm{C}$ within $30 \mathrm{~min}$. In the $\mathrm{HEX}$ channel, IAC was well amplified in the presence of low concentration of target $\left(<10^{3}\right.$ copies), while inhibited in the presence of high concentration of target $\left(>10^{5} \mathrm{cop}-\right.$ ies). Both EV71 and CA16 duplex real time RT-RAA assays were able to detect 10 copies per reaction in the presence of 50 and 100 copies IAC plasmids, respectively. The LoD of EV71and CA16 duplex real time RT-RAA at $95 \%$ probability was 47 copies/reaction and 38 copies/reaction, respectively (Table 2). No crossreactivity was observed with other 170 non-EV71, nonCA16 clinical specimens.

\section{Optimization of the reaction time, analytical sensitivity and specificity of LFS RT-RAA assay}

As shown in Fig. 4a (EV71) and Fig. 5a (CA16), no test line was observed for the $10 \mathrm{~min}$ incubation, and the test line was weakly visible for $20 \mathrm{~min}$ incubation using 100copies plasmid as a template, and no clear difference was shown between $30 \mathrm{~min}$ and $40 \mathrm{~min}$ incubation. Therefore, the reaction time was chosen to be $30 \mathrm{~min}$.

As shown in Fig. $4 \mathrm{~b}$ and Fig. 5b, the results indicated that the sensitivities of the LFS RAA assays for EV71 and CA16 were100 copies per reaction. The detection limits of LFS RT-RAA for EV71 and CA16 were both 90 copies. For the specificity analysis, only EV71 or CA16 was detected by the LFS RT-RAA assays, not other control enteroviruses. These control viruses included coxsackievirus A6 (CA6), coxsackievirus A10 (CA10), coxsackievirusA5 (CA5), coxsackievirus A9 (CA9), coxsackievirus A24 (CA24), coxsackievirus B2 (CB2), coxsackievirus $\mathrm{B} 4$ (CB4), poliovirus 2 (PV2), poliovirus 3 (PV3), echovirus 30 (Eco30), and human enterovirus 14 (HEV14) (Fig. 4c, Fig. 5c)

\section{Evaluation of duplex real time RT-RAA and LFS RT-RAA assays with clinical specimens}

A total of 445 suspected HFMD specimens were used for the clinical evaluation of duplex real time RT-RAA and LFS RT-RAA assays for EV71 and CA16.The RTqPCR assays were carried out simultaneously as parallel tests. RT-qPCR results indicated that 20.4\% (91/445), $22.0 \%$ (98/445), $38.2 \%(170 / 445)$ of the specimens were EV71, CA16 and other enterovirus positive. The remaining 86 samples were RT-qPCR negative, as no enterovirus was identified in these samples.

As shown in the Table 3, with RT-qPCR as the reference assay, the diagnostic sensitivities of duplex real time and LFS RT-RAA assays for EV71 were 92.3, $90.1 \%$, respectively, the diagnostic specificities were both $99.7 \%$, the positive predicative values were both $98.8 \%$, the negative predictive values were 98.1, 97.5\%, respectively. Both assays had high consistency (Kappa values: $0.943,0.929)$. The diagnostic sensitivities of duplex real time and LFS RT-RAA assay for CA16 were 99.0, 94.9\%, respectively, the diagnostic specificities and the positive predicative values were both $100 \%$, the negative predictive values were 99.7, 98.6\%, respectively. Both assays also had high consistency (Kappa values: 0.993, 0.967). As to the samples with discrepant detection results, 7 EV71 specimens and 1 CA16 sample missed by duplex real 


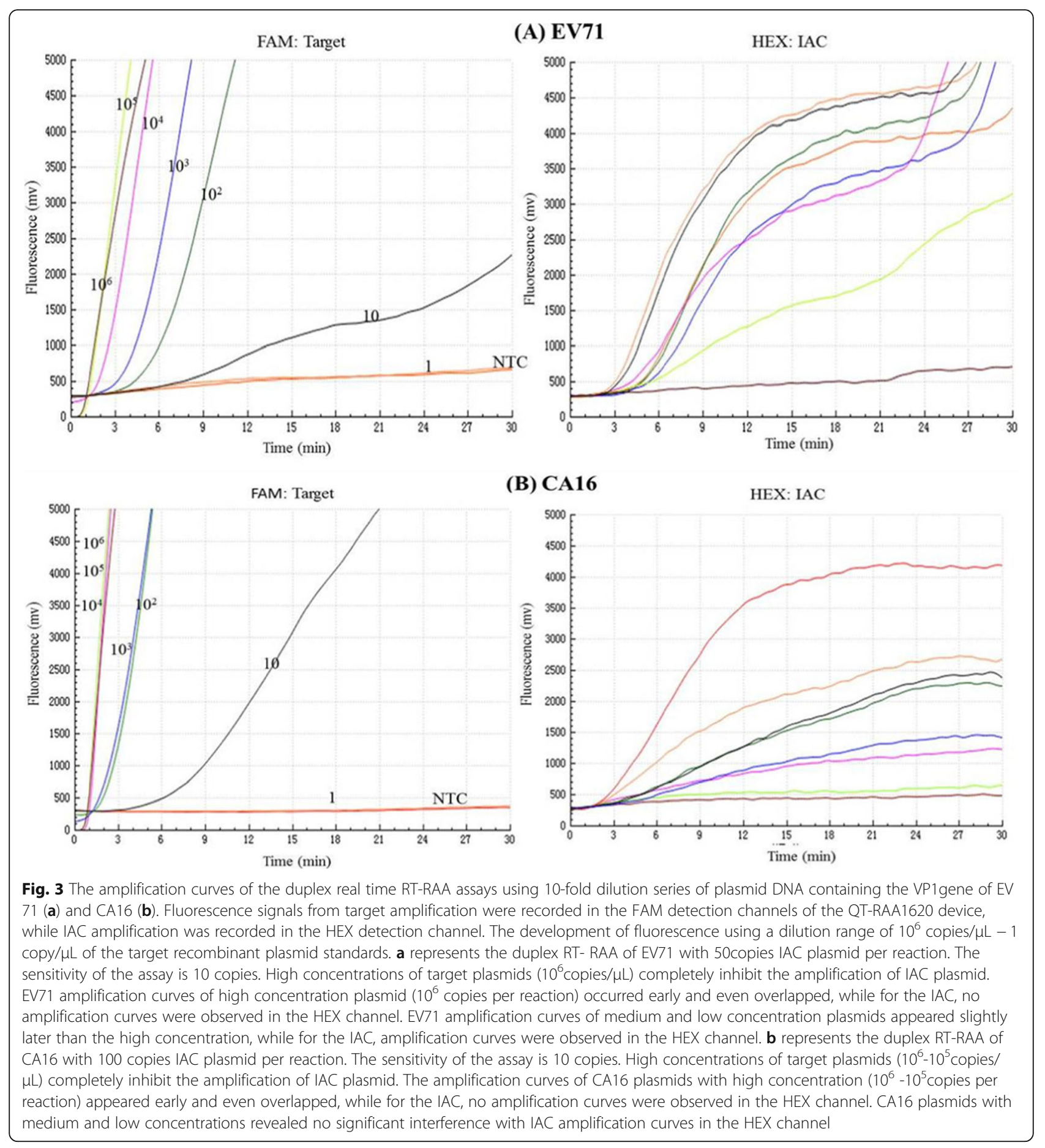

time RT-RAA were found to be positive by RT-qPCR, 9 EV71 specimens and 5 CA16 samples missed by LFS RT-RAA were found to be positive by RT-qPCR. These samples were later confirmed by Sanger sequencing to be true positives. Besides, 1 EV71 positive specimen by duplex real time RT-RAA was tested negative by RTqPCR, which was later confirmed to be true positive by sequencing.

\section{Discussion}

Currently, HFMD is a serious threat to the health of children in China. In the study, we established and evaluated duplex real time RT-RAA and LFS RT-RAA assays for the detection of EV71 and CA16, respectively. In comparison with RT-qPCR, duplex real time RT-RAA assays showed higher diagnostic sensitivities (92.3, 98.9\%) than corresponding LFS RT-RAA assays (90.1, 
Table 2 Assay data used for calculating the detection limit of EV71 and CA16

\begin{tabular}{|c|c|c|c|c|}
\hline \multirow{2}{*}{$\begin{array}{l}\text { Standard } \\
\text { DNA }^{\mathrm{a}} \\
\text { (copies/ } \\
\text { reaction) }\end{array}$} & \multicolumn{2}{|l|}{ EV71pos ${ }^{b}$} & \multicolumn{2}{|l|}{ CA16pos ${ }^{b}$} \\
\hline & Real time RAA & LFS RAA & Real time RAA & LFS RAA \\
\hline $10^{4}$ & $8 / 8$ & $8 / 8$ & $8 / 8$ & $8 / 8$ \\
\hline $10^{3}$ & $8 / 8$ & $8 / 8$ & $8 / 8$ & $8 / 8$ \\
\hline 100 & $8 / 8$ & $8 / 8$ & $8 / 8$ & $8 / 8$ \\
\hline 20 & $5 / 8$ & $0 / 8$ & $6 / 8$ & $0 / 8$ \\
\hline 10 & $3 / 8$ & $0 / 8$ & $4 / 8$ & $0 / 8$ \\
\hline 1 & $0 / 8$ & $0 / 8$ & $0 / 8$ & $0 / 8$ \\
\hline
\end{tabular}

aTenfold serially diluted standard DNA

${ }^{\mathrm{b}}$ The number of positive results per 8 reactions with diluted standard DNA

94.9\%) in detecting EV71 and CA16 respectively. The specificities of two RT-RAA assays were further confirmed by testing 170 other enterovirus RT-qPCRpositive samples. These samples included 57 of CA6, 40 of CA10, which are increasingly prevalent in causing HFMD in China. These results together demonstrated that the proposed methods reveal a high consistency with RT-qPCR method.

Seven EV71 specimens and one CA16 sample were missed by duplex real time RT-RAA, as these sample were with high CT values $(>32)$ by RT-qPCR. Other than the above seven EV71 specimens, two additional EV71 specimens were not detected by LFS RT-RAA. In the case of CA16 specimens, five additional specimens were not detected by LFS RT-RAA. The sequences of RT-RAA primers and probes were subsequently compared with the templates of these samples. Sequence alignment showed that there were 2-3 mismatches occurred in the middle of the forward primer, 1-3 mismatches occurred in the middle of the exo probe. We speculate that sequence variation leads to amplification failure of RT-RAA assay [37]. Previous researches have indicated that the sequences of strains prevalent in different regions were slightly different [38-40], the genotype $\mathrm{C}$, subtype $4 \mathrm{a}$ (C4a) of EV71 and genotype B, subtypes 1a (B1a) and1b (B1b) of CA16 are the major subgenotypes in the mainland of China. Despite this, as the clinical samples in the study were collected from three different cities, one is located in Southern part, one is situated in central part and another in Northern part of China, our results indicated the adaptability of duplex RT-RAA assay for the EV71 and CA16.

We collected different types of specimens in this study, including throat swab specimens $(n=76)$, anal swab specimens $(n=25)$ and stool specimens $(n=344)$, the results showed that there was no significant difference in the detection rate of different specimen types, suggesting the method has very good practicability in testing different types of clinical specimens.

LAMP was previously reported in the detection of EV71 and CA16 [20], however, LAMP assay did not contain an IAC. RPA is increasingly used in agriculture, food safety and pathogen detection [41-45], but few

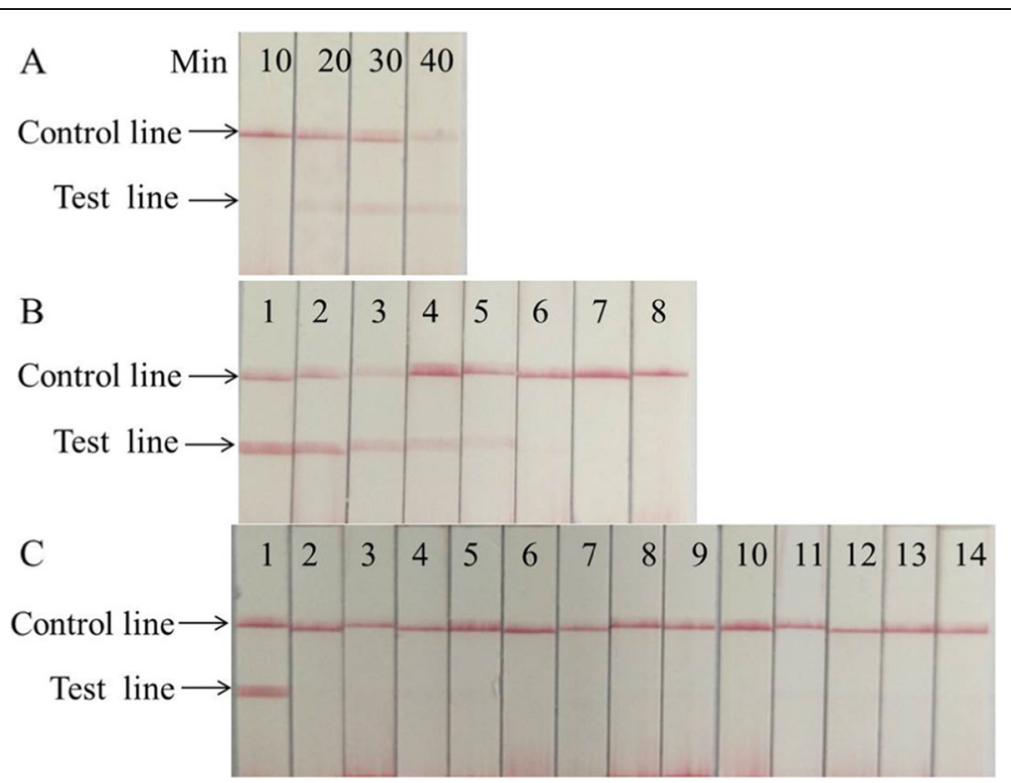

Fig. 4 Performance of EV71 LFS RT-RAA assay. a Optimization experiment of LFS RT-RAA reaction time. When the reaction time was longer than $20 \mathrm{~min}$, the test line was visible. b Analytical sensitivity of the LFS RT- RAA assay. Lane1, $1 \times 10^{6}$ copies, Lane2, $1 \times 10^{5}$ copies, Lane3, $1 \times 10^{4}$ copies, Lane4, $1 \times 10^{3}$ copies, Lane5, $1 \times 10^{2}$ copies, Lane6, $1 \times 10^{1}$ copies, Lane7, 1 copy, Lane8, no template control. c Analytical specificity of the LFS RTRAA assay. Only EV71 specimen was amplified. The other samples were not amplified. Lane1, EV71, Lane2, CA 16, Lane3, CA6, Lane4, CA10, Lane5, CA5, Lane6, CA9, Lane7, CA24, Lane8, CB2, Lane9, CB4, Lane10, PV2, Lane11, PV3, Lane12, Eco30, Lane13, HEV14, Lane14, no template control 


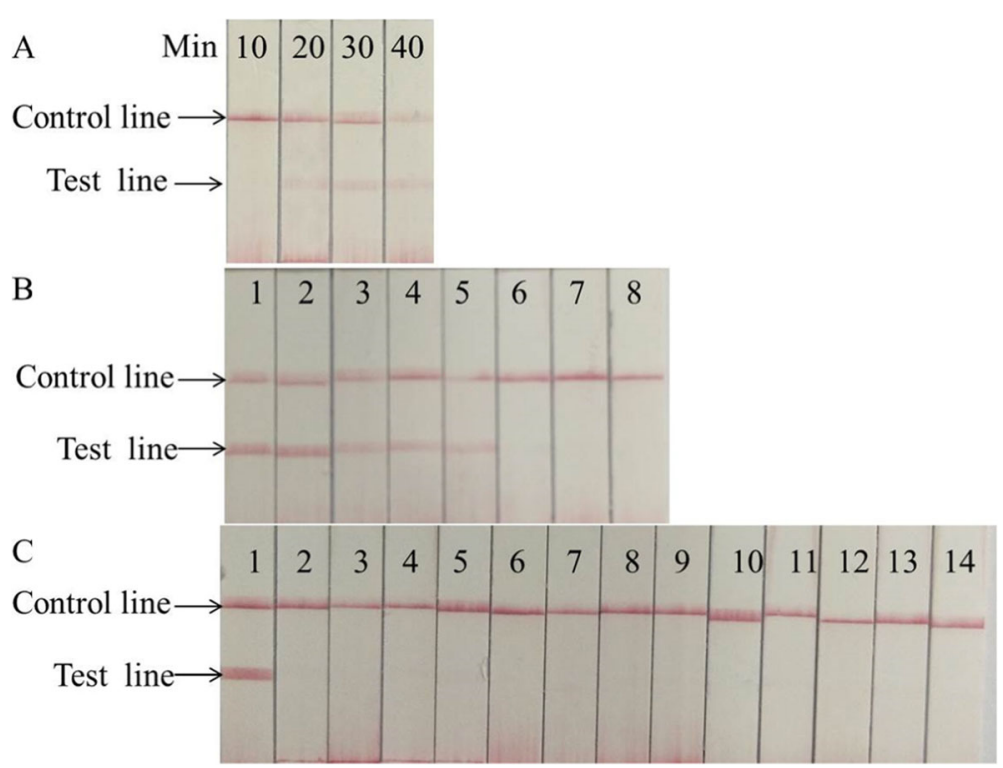

Fig. 5 Performance of CA16 LFS RT-RAA assay. a Optimization experiment of LFS RT-RAA reaction time. When the reaction time was longer than $20 \mathrm{~min}$, the test line was visible. b Analytical sensitivity of the LFS RT-RAA assay. Lane1, $1 \times 10^{6}$ copies, Lane2, $1 \times 10^{5}$ copies, Lane $3,1 \times 10^{4}$ copies, Lane4, $1 \times 10^{3}$ copies, Lane5, $1 \times 10^{2}$ copies, Lane6, $1 \times 10^{1}$ copies, Lane7, 1 copy, Lane8, no template control. c Analytical specificity of the LFS RTRAA assay. Only CA16 specimen was amplified. The other samples were not amplified. Lane1, CA16, Lane2, EV71, Lane3, CA6, Lane4, CA10, Lane5, CA5, Lane6, CA9, Lane7, CA24, Lane8, CB2, Lane9, CB4, Lane10, PV2, Lane11,PV3, Lane12, Eco30, Lane13, HEV14, Lane14, no template control

Table 3 Comparison of clinical evaluation of two RT-RAA assays and RT-qPCR assay to detect EV71 and CA16

\begin{tabular}{|c|c|c|c|c|}
\hline \multirow[t]{2}{*}{ RT- $\mathrm{PPCR}$} & \multicolumn{2}{|c|}{$\begin{array}{l}\text { Duplex real time RT- } \\
\text { RAA }\end{array}$} & \multicolumn{2}{|c|}{ LFS RT-RAA } \\
\hline & Positive & Negative & Positive & Negative \\
\hline \multicolumn{5}{|l|}{$\overline{\text { EV71 }}$} \\
\hline Positive & 84 & 7 & 82 & 9 \\
\hline Negative & 1 & 353 & 1 & 353 \\
\hline $\begin{array}{l}\text { Diagnostic sensitivity } \\
(\%)\end{array}$ & 92.3 & & 90.1 & \\
\hline $\begin{array}{l}\text { Diagnostic specificity } \\
(\%)\end{array}$ & & 99.7 & & 99.7 \\
\hline PPV (\%) & 98.8 & & 98.8 & \\
\hline NPV (\%) & & 98.1 & & 97.5 \\
\hline Kappa & 0.943 & & 0.929 & \\
\hline \multicolumn{5}{|l|}{ CA16 } \\
\hline Positive & 97 & 1 & 93 & 5 \\
\hline Negative & 0 & 347 & 0 & 347 \\
\hline $\begin{array}{l}\text { Diagnostic sensitivity } \\
(\%)\end{array}$ & 99.0 & & 94.9 & \\
\hline $\begin{array}{l}\text { Diagnostic specificity } \\
(\%)\end{array}$ & & 100 & & 100 \\
\hline PPV (\%) & 100 & & 100 & \\
\hline NPV (\%) & & 99.7 & & 98.6 \\
\hline Kappa & 0.993 & & 0.967 & \\
\hline
\end{tabular}

PPV Positive predictive value, NPV Negative predictive value publications reported duplex real time RPA assay containing IAC. Dan Yin [22] reported a rapid RT-RPA assay to detect EV71. The 95\% detection limit was 3.767 $\log 10$ genomic copies (LGC)/reaction, with100\% specificity, but no IAC was included in the assay. In our study, the introduction of IAC effectively eliminated false negative results or invalid results. Two strategies are used to design IAC: one is a noncompetitive system, the other is competitive system [46-49]. A noncompetitive IAC system contains 2 pairs of primers to amplify the target DNA and control DNA, respectively. The shortcoming of a noncompetitive IAC is that it might reduce amplification efficiency for target because of the introduction and interference of control primers with target primers. In the case of competitive strategy, one set of common primers is used to amplify both the target DNA and the IAC, which eliminates the risk of interference among multiple pairs of primers. By optimizing the amount of primers, the ratio of target probes and IAC probes, and the amount of IAC plasmids, the negative impact of IAC on the detection sensitivity of target could be minimized.

Although same primers and probes were used for the amplification, the LFS RT-RAA assay was less sensitive than duplex real time RT-RAA assay. As LFS RT-RAA assay is to detect the double-labeled amplicons generated by extension of a small portion of the amplification product driven by the post-cleavage LF probe and 
reverse primer, this may influence the sensitivity of the LFS RT-RAA assay. Another influence factor might be due to the detection principle of lateral flow strip. The minimum number of molecules that can be detected on the test strip would also affect the final test results. While the detection of real time RT-RAA mainly depends on the fluorescence device and analyze software, results are more accurate and sensitive.

The duplex real time RT-RAA assay can be completed in a single tube in one step at $42{ }^{\circ} \mathrm{C}$ within $30 \mathrm{~min}$ without complicated operations or expensive equipment compared to traditional real time PCR. Additionally, introduction of the IAC effectively avoided the appearance of false negatives and invalid results. Use of the B6100 Oscillation mixer helped to further reduce manual error and improve repeatability of experimental results. Although the sensitivity of LFS RT-RAA is slightly lower than the sensitivity of duplex real-time RT-RAA, equipment-free and visual detection make the LFS RTRAA well suitable for on-site screening in resourcelimited areas. There are still some limitations in this study such as slightly lower sensitivity of duplex real time RT-RAA assay and lack of IAC in LFS RT-RAA assay. For improved practicability for point-of-care testing in resource poor clinical settings, the sensitivities of both RT-RAA assays need to be further increased to meet the requirement of initial screening while maintaining appropriate specificity. Future study will also include attempting the direct RT-RAA detection without RNA extraction and clinical evaluation using large sample size.

\section{Conclusion}

In summary, the study demonstrated that the duplex real time RT-RAA assay is rapid and sensitive enough to detect EV71 and CA16 from clinical specimens and LFS RT-RAA assay is potentially suitable for field use in primary clinical settings.

\section{Abbreviations}

CA10: Coxsackievirus A10; CA16: Coxsackievirus A16; CA24: Coxsackievirus A24; CA5: Coxsackievirus A5; CA6: Coxsackievirus A6; CA9: Coxsackievirus A9; CB2: Coxsackievirus B2; CB4: Coxsackievirus B4; Eco30: Echovirus 30;

EV71: Enterovirus 71; HEV14: Human enterovirus 14; HFMD: Hand, Foot and Mouth Disease; IAC: Internal amplification controls; LAMP: Loop mediated isothermal amplification; LFS: Lateral flow strip; LoD: The limit of detection; NPV: Negative predictive value; PPV: Positive predictive value; PV2: Poliovirus 2; PV3: Poliovirus 3; qPCR: Quantitative PCR; RPA: Recombinase polymerase amplification; RT-qPCR: Reverse transcription-quantitative PCR; RTRAA: Reverse-transcription recombinase aided amplification

\section{Acknowledgements}

We thank Shandong, Hebei, and Hunan centers for disease control and prevention for providing the clinical samples.

\section{Authors' contributions}

$X N-L, X X-S, M H-L, Y-X$, and XJ-M conceived and designed the experiments; $X N-L, J J-Q$ and RH-W performed the experiments; RQ-Z, QX-D and T-F collected the samples and data; GH-F and XD-B analyzed the data; XN-L and XJ-
M wrote the paper; $X N-L, X X-S, M H-L, Y-X$ and $X J-M$ reviewed and revised the manuscript. All authors have contributed to the interpretation and discussion of the results, and approved the final version of the manuscript for publication.

\section{Funding}

This work was funded by grants from the China Mega-Projects for Infectious Disease (2018ZX10711001, 2017ZX10302301-004-002, 2017ZX10104001 and 2018ZX10713-002) and IVDC (2019HYDQNJJ03).

\section{Availability of data and materials}

The datasets used and analyzed during the current study are included within this article and in additional file.

\section{Ethics approval and consent to participate}

The work was approved by the Institutional Review Boards of National Institute for Viral Disease Control and Prevention, Center for Disease Control and Prevention of China. All participants have given verbal informed consent.

\section{Consent for publication}

Not applicable.

\section{Competing interests}

The authors declare that they have no competing interests.

Received: 14 June 2019 Accepted: 2 December 2019

Published online: 30 December 2019

\section{References}

1. Liu W, et al. Co-circulation and genomic recombination of coxsackievirus A16 and enterovirus 71 during a large outbreak of hand, foot, and mouth disease in Central China. PLoS One. 2014;9(4):e96051.

2. Kashyap S, Verma GK. Hand-foot-mouth disease: outbreak in Shimla. Indian Pediatr. 2014;51(2):155.

3. Duong $V$, et al. Molecular epidemiology of human enterovirus 71 at the origin of an epidemic of fatal hand, foot and mouth disease cases in Cambodia. Emerg Microbes Infect. 2016;5(9):e104.

4. Chua KB, Kasri AR. Hand foot and mouth disease due to enterovirus 71 in Malaysia. Virol Sin. 2011;26(4):221-8

5. Cabrerizo M, et al. Molecular epidemiology of enterovirus 71, coxsackievirus A16 and A6 associated with hand, foot and mouth disease in Spain. Clin Microbiol Infect. 2014;20(3):0150-6.

6. Shah VA, et al. Clinical characteristics of an outbreak of hand, foot and mouth disease in Singapore. Ann Acad Med Singap. 2003;32(3):381-7.

7. Long $L$, et al. Neurological complications and risk factors of cardiopulmonary failure of EV-A71-related hand, foot and mouth disease. Sci Rep. 2016;6:23444.

8. Zhang YC, et al. Clinical characteristics and treatment of severe encephalitis associated with neurogenic pulmonary edema caused by enterovirus 71 in China. World J Emerg Med. 2010;1(2):108-13.

9. $\mathrm{Yu} \mathrm{N}$, et al. Evaluation of human enterovirus 71 and coxsackievirus A16 specific immunoglobulin $\mathrm{M}$ antibodies for diagnosis of hand-foot-andmouth disease. Virol J. 2012;9:12.

10. Xu F, et al. Development of an IgM-capture ELISA for Coxsackievirus A16 infection. J Virol Methods. 2011;171(1):107-10.

11. Cui A, et al. The development and application of the two real-time RT-PCR assays to detect the pathogen of HFMD. PLoS One. 2013;8(4):e61451.

12. Dubot-Peres $A$, et al. SYBR green real-time PCR for the detection of all enterovirus-A71 genogroups. PLoS One. 2014;9(3):e89963.

13. Niu P, et al. Development of a highly sensitive real-time nested RT-PCR assay in a single closed tube for detection of enterovirus 71 in hand, foot, and mouth disease. Arch Virol. 2016;161(11):3003-10.

14. Puenpa J, et al. Development of single-step multiplex real-time RT-PCR assays for rapid diagnosis of enterovirus 71, coxsackievirus A6, and A16 in patients with hand, foot, and mouth disease. J Virol Methods. 2017;248:92-9.

15. Tan EL, et al. Rapid detection of enterovirus 71 by real-time TaqMan RT-PCR J Clin Virol. 2008;42(2):203-6.

16. Thanh $\Pi$, et al. Validation and utilization of an internally controlled multiplex real-time RT-PCR assay for simultaneous detection of 
enteroviruses and enterovirus A71 associated with hand foot and mouth disease. Virol J. 2015;12:85.

17. Zhang $\mathrm{S}$, et al. A one-step, triplex, real-time RT-PCR assay for the simultaneous detection of enterovirus 71, coxsackie A16 and panenterovirus in a single tube. PLoS One. 2014;9(7):e102724.

18. Casper ET, et al. Development and evaluation of a method to detect and quantify enteroviruses using NASBA and internal control RNA (IC-NASBA). J Virol Methods. 2005;124(1-2):149-55.

19. Romano JW, et al. NASBA technology: isothermal RNA amplification in qualitative and quantitative diagnostics. Immunol Investig. 1997;26(1-2):15-28.

20. Nie K, et al. Visual detection of human enterovirus 71 subgenotype C4 and Coxsackievirus A16 by reverse transcription loop-mediated isothermal amplification with the hydroxynaphthol blue dye. J Virol Methods. 2011; 175(2):283-6.

21. Wang $X$, et al. Detection of enterovirus 71 using reverse transcription loopmediated isothermal amplification (RT-LAMP). J Virol Methods. 2012;179(2):330-4.

22. Yin $\mathrm{D}$, et al. Development and evaluation of a rapid recombinase polymerase amplification assay for the detection of human enterovirus 71 . Arch Virol. 2018;163(9):2459-63.

23. Wu Y, et al. Rapid and visual detection of Lawsonia intracellularis with an improved recombinase polymerase amplification assay combined with a lateral flow dipstick. BMC Vet Res. 2019;15(1):97.

24. Miao F, et al. Rapid and sensitive Recombinase polymerase amplification combined with lateral flow strip for detecting African swine fever virus. Front Microbiol. 2019;10:1004.

25. Zhang TT, et al. Rapid and simple detection of Glaesserella parasuis in synovial fluid by recombinase polymerase amplification and lateral flow strip. BMC Vet Res. 2019;15(1):294.

26. Jaroenram $W$, Owens $L$. Recombinase polymerase amplification combined with a lateral flow dipstick for discriminating between infectious Penaeus stylirostris densovirus and virus-related sequences in shrimp genome. J Virol Methods. 2014;208:144-51.

27. Zhang X, et al. Rapid detection of Salmonella with Recombinase aided amplification. J Microbiol Methods. 2017;139:202-4.

28. Chen C, et al. Use of a rapid reverse-transcription recombinase aided amplification assay for respiratory syncytial virus detection. Diagn Microbiol Infect Dis. 2018;90(2):90-5.

29. Shen $X X$, et al. A rapid and sensitive recombinase aided amplification assay to detect hepatitis B virus without DNA extraction. BMC Infect Dis. 2019;19(1):229.

30. Yan TF, et al. Development of a reverse transcription recombinase-aided amplification assay for the detection of coxsackievirus A10 and coxsackievirus A6 RNA. Arch Virol. 2018;163(6):1455-61.

31. China, T.M.o.H.o.t.P.s.R.o. Guidelines for the diagnosis and treatment of hand, foot and mouth disease (2010 edition). 2010 2010.4.20]; Available from: http://www.nhc.gov.cn/yzygj/s3593g/201306/6d935c0f43cd4a1fb46f8f71 acf8e245.shtml.

32. Babu B, et al. A field based detection method for rose rosette virus using isothermal probe-based reverse transcription-recombinase polymerase amplification assay. J Virol Methods. 2017;247:81-90.

33. Li J, Macdonald J, von Stetten F. Review: a comprehensive summary of a decade development of the recombinase polymerase amplification. Analyst. 2018;144(1):31-67.

34. Han J, et al. Long persistence of EV71 specific nucleotides in respiratory and feces samples of the patients with hand-foot-mouth disease after recovery. BMC Infect Dis. 2010;10:178.

35. Lalkhen AG, McCluskey A. Clinical tests: sensitivity and specificity. Contin Educ Anaesth Crit Care Pain. 2008;8(6):221-3.

36. van Stralen KJ, et al. Diagnostic methods I: sensitivity, specificity, and other measures of accuracy. Kidney Int. 2009;75(12):1257-63.

37. Daher RK, et al. Influence of sequence mismatches on the specificity of recombinase polymerase amplification technology. Mol Cell Probes. 2015;29(2):116-21.

38. Huang J, et al. Epidemiology of Recurrent Hand, Foot and Mouth Disease, China, 2008-2015. Emerg Infect Dis. 2018;24(3):432-42.

39. Huang $X$, et al. Epidemiological and etiological characteristics of hand, foot, and mouth disease in Henan, China, 2008-2013. Sci Rep. 2015;5:8904.

40. Zhang J, et al. Statistical monitoring of the hand, foot and mouth disease in China. Biometrics. 2015;71(3):841-50.
41. Abd El Wahed A, et al. Recombinase Polymerase Amplification Assay for Rapid Diagnostics of Dengue Infection. PLoS One. 2015;10(6):e0129682.

42. Castellanos-Gonzalez A, et al. Molecular diagnosis of protozoan parasites by Recombinase polymerase amplification. Acta Trop. 2018;182:4-11.

43. Du XJ, et al. Recombinase polymerase amplification combined with lateral flow strip for listeria monocytogenes detection in food. J Food Sci. 2018;83(4):1041-7

44. Gao W, et al. Recombinase polymerase amplification combined with lateral flow dipstick for equipment-free detection of Salmonella in shellfish. Bioprocess Biosyst Eng. 2018;41(5):603-11.

45. Raja B, et al. Development of a panel of recombinase polymerase amplification assays for detection of common bacterial urinary tract infection pathogens. J Appl Microbiol. 2017;123(2):544-55.

46. Akbarian A, et al. Designing novel and simple competitive internal amplification control for reliable PCR diagnosis of herpes simplex virus. Jundishapur J Microbiol. 2015;8(2):e16260.

47. Chen $\mathrm{Q}$, et al. Development and evaluation of a real-time method of simultaneous amplification and testing of enterovirus 71 incorporating a RNA internal control system. J Virol Methods. 2014;196:139-44.

48. Higgins $\mathrm{O}$, et al. Duplex recombinase polymerase amplification assays incorporating competitive internal controls for bacterial meningitis detection. Anal Biochem. 2018;546:10-6.

49. Pionzio AM, McCord BR. The effect of internal control sequence and length on the response to PCR inhibition in real-time PCR quantitation. Forensic Sci Int Genet. 2014;9:55-60.

\section{Publisher's Note}

Springer Nature remains neutral with regard to jurisdictional claims in published maps and institutional affiliations.

\section{Ready to submit your research? Choose BMC and benefit from:}

- fast, convenient online submission

- thorough peer review by experienced researchers in your field

- rapid publication on acceptance

- support for research data, including large and complex data types

- gold Open Access which fosters wider collaboration and increased citations

- maximum visibility for your research: over $100 \mathrm{M}$ website views per year

At BMC, research is always in progress.

Learn more biomedcentral.com/submissions 